\title{
The role of comparative panoramic visualization of radiographs in the diagnosis of developmental dysplasia of the hip: reply to Daza and Osorio
}

\author{
Marc S. Keller • Els L. F. Nijs
}

Received: 23 September 2009 / Accepted: 23 September 2009/Published online: 24 November 2009

(C) Springer-Verlag 2009

Sir,

We read with interest the recent letter of Drs. Daza and Osorio [1] concerning radiography in developmental dysplasia of the hip (DDH) and are pleased to note their comments and respond to them. We are in full agreement about their depiction of findings in DDH and their explanation of the correlation with normal and abnormal stresses upon the developing neonatal and infant acetabulum. When one examines the literature and history of medical care of $\mathrm{DDH}$, one finds many methods over the years that have been developed radiographically to examine qualitatively and quantitatively the hip joints of infants and children. All are helpful, with some more than others.

Our recent article was published in a supplement of Pediatric Radiology that summarized our contribution to the postgraduate course at the 2008 annual meeting of the Society for Pediatric Radiology where the theme was "Evidence-Based Imaging in Pediatrics." Our task was to examine the last decade of literature on imaging in DDH and report upon the latest available information of whether outcomes in babies with DDH are improved by imaging. In doing so, we reviewed all modalities and comparisons with clinical examination that we could find. Admittedly, we searched only in English.

The development and evolution of hip sonography in the last 20 years has been in response to the late emergence of DDH that previously had not been prevented by clinical examination alone or the accompanying use of radiography.

\footnotetext{
M. S. Keller $(\bowtie) \cdot$ E. L. F. Nijs

Department of Radiology,

The Children's Hospital of Philadelphia,

34 Street and Civic Center Blvd,

Philadelphia, PA 19104, USA

e-mail: kellerm@email.chop.edu
}

In our opinion, the best evidence, as reported in our article, has come out of two Norwegian randomized controlled trials $[2,3]$. In babies without risk factors, no statistical difference has been found between expert clinical hip examiners and expert hip sonologists in preventing the rare emergence of cases of late DDH. In babies with DDH risk factors, there has been a trend, not statistically significant, of hip sonography being slightly better diagnostically than clinical examination alone. Some evidence and opinion suggest that when clinical examiners are inexperienced or are unsure of their findings, the use of imaging is likely to be more sensitive in detecting DDH or confirming normal.

We are not aware of any large-scale recent trials of infant AP pelvic radiography being compared to clinical hip examination and then checking long-term outcome and the late discovery of DDH. In our opinion, selective use of hip radiography remains a useful adjunct in the care of children beyond early infancy in the follow-up of DDH but has not been shown to improve outcomes when used in routine neonatal and early infant screening.

\section{References}

1. Daza NL, Osorio L (2009) The role of comparative panoramic visualization of radiographs in the diagnosis of developmental dysplasia of the hip. Pediatr Radiol 39: doi 10.1007/s00247-0091427-y

2. Holen KJ, Tegnander A, Bredland $\mathrm{T}$ et al (2002) Universal or selective screening of the neonatal hip using ultrasound? A prospective, randomized trial of 15, 529 newborn infants. J Bone Joint Surg Br 84:886-890

3. Rosendahl K, Markestad T, Lie RT (1994) Ultrasound screening for developmental dysplasia of the hip in the neonate: the effect on treatment rate and prevalence of late cases. Pediatrics 94:47-52 